\title{
Abetalipoproteinemia - A Rare Disease Presenting as Bleeding Disorder
}

\author{
Geeta Vidyadharan ${ }^{1}$, Bitty Kurian², Suchitra Sivadas 3 , Sheela Nampoothiri' ${ }^{4}$, Jyotsna Yesodharan ${ }^{5}$ \\ 1,2,5 Department of Pathology, Amrita Institute of Medical Sciences, Kochi, Kerala, India. \\ ${ }^{3}$ Department of Paediatrics, Amrita Institute of Medical Sciences, Kochi, Kerala, India. \\ ${ }^{4}$ Department of Paediatric Genetics, Amrita Institute of Medical Sciences, Kochi, Kerala, India.
}

\section{PRESENTATION OF CASE}

A 5-month-old child presented to the Paediatric Outpatient Department with complaints of fever, loose stools, blood in stool, and multiple subcutaneous swellings over the chest wall. The patient was given antibiotics and treated for acute gastroenteritis with a possibility of component of cow's milk allergy also being considered. The routine blood counts revealed platelet count of $4.94 \mathrm{~K} / \mu \mathrm{L}$, along with Hb 10.2 g / dL, MCV - 80 fL, WBC - 13.6 x 109 / L. An LDH of 512 U / L (normal up to $451 \mathrm{U} / \mathrm{L}$ ) and ESR of $2 \mathrm{~mm} / 1^{\text {st }} \mathrm{hr}$. Liver function tests were within normal range. Her preliminary coagulation screening test showed both PT as well as aPTT to be prolonged beyond $120 \mathrm{sec}$ each. She was further sent for complete laboratory evaluation for bleeding disorder. In view of the history of foul smelling stools, suggesting malabsorption, stool sample was analysed which showed presence of fat globules. USG was reported as normal study. Blood group was 0 positive.

Abetalipoproteinemia is a very rare metabolic, autosomal recessive disease resulting from mutations encoding microsomal triglyceride transfer protein (MTP) leading to deficiencies in the apolipoproteins B-48 and B-100 with reported prevalence of less than one case per 100,000. Typical manifestations are failure to thrive, hypocholesterolaemia and fat malabsorption. Other features like fatty liver, acanthocytosis, and anaemia are usually present in infancy and neuro-ocular complications during adolescence. Early diagnosis and management can prevent disease progression.

\section{CLINICAL DIAGNOSIS}

Malabsorption syndrome.

\section{PATHOLOGICAL DISCUSSION}

We received the patient in the haemostasis laboratory as a part of complete workup for bleeding disorder. We took detailed history with respect to bleeding symptoms, which provided history of passing blood in stools from the age of 3 months. Also there was history of recent onset of subcutaneous swellings over chest and hands. There was no history of bleeding from other orifices or any evidence or complaints of skin bleeds or joint bleeds or any positive family history. The mother did not give history of any excessive bleeding during menstruation either which would have gone in favour of the more common inherited mild bleeding disorders like Von Willebrand disease.
Corresponding Author: Dr. Geeta Vidyadharan, Associate Professor, Department of Pathology, Amrita Institute of Medical Sciences \& Research Centre, Amrita University, Ponekkara, Cochin-682041, Kerala, India. E-mail: keerthanageeta@gmail.com

DOI: $10.14260 /$ jemds/2020/670

How to Cite This Article:

Vidyadharan G, Kurian B, Sivadas $S$, et al, Abetalipoproteinemia - a rare disease presenting as bleeding disorder. J. Evolution Med. Dent. Sci. 2020;9(41):3062-3064, DOI: $10.14260 / \mathrm{jemds} / 2020 / 670$

Submission 28-04-2020,

Peer Review 29-06-2020,

Acceptance 06-07-2020,

Published 12-10-2020.

Copyright (C) 2020 JEMDS. This is an open access article distributed under Creative Commons Attribution License [Attribution 4.0 International (CC BY 4.0)] 
On examination the baby appeared to be listless and small for age. Feeding history revealed recurrent vomiting and passing of frothy, foul smelling stools. Meanwhile the haemoglobin further dropped to $7.7 \mathrm{~g} / \mathrm{dL}$ in about a month's time, however with maintained WBCs and platelets which was indicative of possibility of on-going bleed. Her stool tested positive for blood (Non-Benzidine Kit for occult blood).

Her preliminary coagulation screening test showed prolongation of both PT and aPTT (> $120 \mathrm{sec}$ ) TT $-16.3 \mathrm{sec}$ (17.3 sec), Fibrinogen - $226 \mathrm{mg} / \mathrm{dL}(200-400 \mathrm{mg} / \mathrm{dL})$ Mixing studies showed correction of both PT and aPTT value. These values were indicative of factor deficiency pattern, especially that involving a factor / s participating in intrinsic as well as extrinsic pathways. The complete correction of the PT and aPTT value was noted on mixing with pooled normal plasma eliminating the suspicion of an acquired inhibitor blocking coagulation factors.

Mixing studies were also carried out with aged and adsorbed plasma (freshly prepared) samples exhibiting correction with aged sera, which pointed toward deficiencies of either factors V, VII, IX or X.

When the patient reported to us for workup, her weight had reduced further from $4.07 \mathrm{Kg}$ to $3.5 \mathrm{Kg}$ and was given vitamin $\mathrm{K}$ orally for 3 days, after which her subcutaneous swelling had disappeared. The preliminary panel for coagulation revealed PT - $13.1 \mathrm{sec}(14.3 \mathrm{sec})$, aPTT - $32.0 \mathrm{sec}$ (32.2 sec), which were normal suggesting improvement of PT and aPTT following Vitamin K administration.

Further Vitamin $\mathrm{K}$ dependent clotting factor assays were performed by clot based method for the evaluation of factors. The investigations revealed Factor II - $76 \%$ (Reference Range: 62 - $103 \%$ ), Factor VII-123 \% (Reference Range: 83 - $160 \%$ ), Factor IX - 67\% (Reference Range: 43 - $121 \%$ ), Factor X - 80 $\%$ (Reference Range: 77 - $122 \%$ ). The factor especially II and IX were of borderline levels. Other factors were within normal limits. Since all of these factors are usually seen to be reduced commonly in conditions affecting liver, we reviewed the liver function tests which were noted to be normal including Serum Bilirubin, SGOT, SGPT, Alkaline phosphatase. Meanwhile as part of protocol for coagulation work up, peripheral smear done for platelet assessment showed prominent acanthocytes in more than $80 \%$ of the RBCs. (Figure 1)

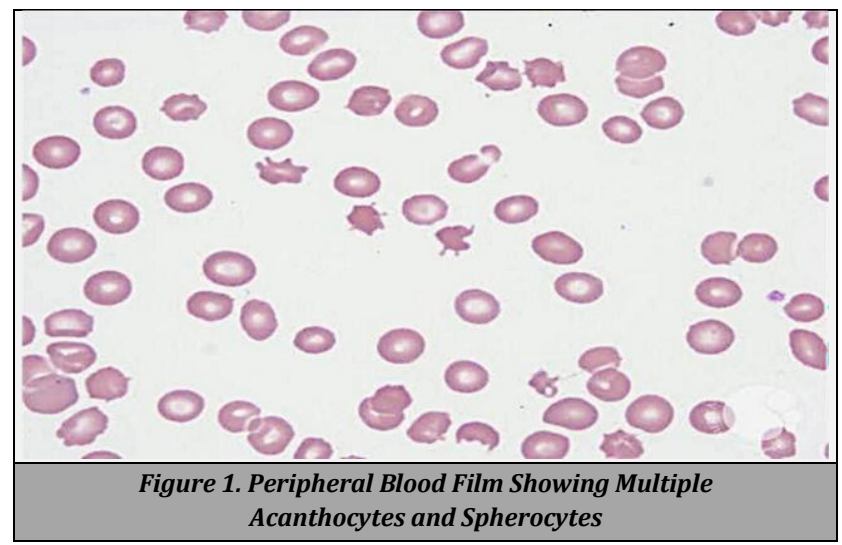

Putting together, findings of a listless, sick looking child of 7 months age presenting with history of fever, diarrhoea, symptoms of malabsorption with evidence of blood in stools and subcutaneous swellings, having acanthocytes in peripheral blood, low ESR, deranged PT / aPTT and exhibiting
Vitamin $\mathrm{K}$ dependent coagulation factor deficiency pattern, leading us to suspect abetalipoproteinemia. Subsequently the sample was sent for Lipid profile study which showed very low value for triglycerides $10.4 \mathrm{mg} / \mathrm{dL}$ (34 - $74 \mathrm{mg} / \mathrm{dL}$ ), VLDL - $2.1 \mathrm{mg} / \mathrm{dL}$ (0 - $30 \mathrm{mg} / \mathrm{dL}$ ), HDL - $17.3 \mathrm{mg} / \mathrm{dL}$ (> $40 \mathrm{mg} /$ $\mathrm{dL}$ ) Vitamin E level was very low, $<2.15 \mathrm{mcg} / \mathrm{mL}(3.0-9.0$ $\mathrm{mcg} / \mathrm{mL}$ ).

The sample of the proband was sent for genetic studies, which revealed presence of homozygous mutation Exon 14-c. 2064dupC (p.Glu689ArgfsTer10) of MTTP gene confirming abetalipoproteinemia.

\section{DISCUSSION OF MANAGEMENT}

Early diagnosis of the uncommon entity helped in timely intervention regarding diet modifications and genetic counselling.

The patient is being treated with supplementations (Vitamin K, 5 mg IV, Vitamin A, uprise D3, Vitamin E - 400 mg, MCT oil-based fat) and was put on fat restricted diet. Currently the patient is doing well, milestones reached up to 1 year 4 months, improved weight gain to $6.2 \mathrm{Kg}$. The patient is on regular Vitamin supplements.

Abetalipoproteinemia (ABL) or otherwise known as Bassen-Kornzweig Syndrome is a rare genetic disease inherited as autosomal recessive disorder, leading to markedly low levels of triglycerides in plasma along with low cholesterol and fat soluble vitamins leading to various manifestations. ${ }^{1}$ It has a reported prevalence of less than one case per 100,000, and results from mutations in the gene coding for microsomal triglyceride transfer protein (MTP). Therefore malabsorption of fat soluble vitamins leads to hepatic, neurologic and ophthalmologic effects in the form of spinocerebellar degeneration, coagulation disorder and pigmented retinopathy and acanthocytes in blood. ${ }^{2}$

Bassen and Kornzweig first described this disease when describing a child born to consanguineous couple appearing similar to Friedreich's ataxia with an atypical type of retinitis pigmentosa. ${ }^{3}$ The disorder was named "abetalipoproteinemia" by Salt and colleagues later. Almost all of the patients diagnosed as abetalipoproteinemia have gastrointestinal symptoms include steatorrhoea, vomiting and failure to thrive. Endoscopy if performed would reveal typical gelee blanche / white hoar frosting appearance, and the corresponding microscopy show lipid laden enterocytes. In the liver steatosis, hepatomegaly, and elevated serum transaminases may be noted. ${ }^{1}$

In ABL patients, the fat soluble vitamin especially vitamin $\mathrm{E}$ are seen to be markedly low though vitamins $\mathrm{A}, \mathrm{D}$, and $\mathrm{K}$ levels are not so significantly affected. This could be due to the fact that vitamin E predominantly relies on Apo-B mediated intestinal absorption ${ }^{1}$. Most of the vitamin $\mathrm{E}$ in intestinal cells gets incorporated into chylomicrons. However vitamin $D$ is not dependent on lipoproteins for their absorption although vitamin $\mathrm{A}$ and $\mathrm{K}$ initially follow the journey through intestine and liver via the conventional lipoprotein way, subsequently they have their independent transportation systems in the circulation.

Vitamin E deficiency is, thus, a chief factor in the pathogenesis of ABL. Vitamin $\mathrm{E}$ is a free radical scavenger and 
protects the mitochondrial membranes, therefore in its deficiency, lipoperoxidation leads to the formation of pigment and these deposits in multiple organ tissues like skeletal striated muscle, liver, myocardium, spinal cord, even involving neurological system. Although few ABL patients have had developmental delays or intellectual disabilities intelligence is usually unaffected in ABL. The reduced plasma lipid levels change the structural integrity of retina and RBCs causing retinopathy as well as abnormally shaped red cells, low ESR, Vitamin E deficiency could be the cause of haemolytic anaemia also. ${ }^{1}$ Coagulation abnormalities are due to reduced levels of coagulation factors. Cardiac muscle myopathy / neuropathy or lipofuscin deposition affect these organs function. GI tracts as well as neurological malignancies have been described in ABL patients. ${ }^{1}$

Demonstrations of severe hypobetalipoproteinaemia in children whose parents have normal lipid profile suggest a diagnosis of ABL and guide the laboratory for analysis of the MTP gene. If parents have hypobetalipoproteinaemia, first consideration to be given to conditions like homozygous FHBL, a condition that suggests the involvement of other candidate genes (i.e. APOB or PCSK9). 4

Also important to note that the various reviewed literature have suggested that the prognostic factors predicting disease course include age at diagnosis, onset of treatment with low fat diet and vitamin replacement therapy, type of MTP mutation and APOE genotype with the long-term outcome of patients with ABL. ${ }^{5}$

The current standard treatment for $\mathrm{ABL}$ is dietary modification and replacement of fat soluble vitamins. A low fat diet has been shown to improve steatorrhea associated with fat malabsorption and allow absorption of other nutrients essential for growth and development. Oral vitamin E is typically given in daily doses ranging from 2400 to 12000 IU. In one study, serum vitamin $\mathrm{E}$ concentrations, which were initially undetectable in all ABL patients, became measurable after oral supplementation, although they never reached the normal range. Vitamin D deficiency is not consistently described among ABL patients. However, low serum ionized calcium, vitamin $\mathrm{D}$ and bony abnormalities have been described, and thus vitamin D replacement (1000 mg daily) should be considered in all ABL patients. An abnormal coagulation profile with prolonged prothrombin time and increased international normalized ratio has been reported in many ABL patients: two patients had severe gastrointestinal bleeding. In addition vitamin $\mathrm{E}$ absorption can exacerbate the deficit in vitamin $\mathrm{K}$ therefore, replacement of vitamin $\mathrm{K}$ is necessary ( $5 \mathrm{mg}$ daily). Other supplementary nutrients like iron, folic acid can also be considered. Patients need to be followed regularly for evaluation of symptoms and complications, and to monitor compliance with therapy.5 Early diagnosis and early planned protocols are essential to prevent or slow down the progression of manifestation following fatsoluble vitamins deficiencies.

In summary, $\mathrm{ABL}$ is a rare disease of lipoprotein metabolism that has drawn attention to the importance of MTP in the assembly and secretion of apo-B containing lipoproteins.

Without treatment, ABL symptoms can be debilitating in most of the patients and life expectancy is expected to be reduced. Current evidence suggests that early treatment with high oral doses of combined vitamin $\mathrm{A}$ and $\mathrm{E}$, if introduced early enough, can reduce the potential severity of neuropathy and retinopathy. There is still a need for novel therapeutic approaches to $\mathrm{ABL}$, since vitamin therapy alone is not sufficient to completely control or cure this disease. ${ }^{5}$

\section{FINAL DIAGNOSIS}

Abetalipoproteinemia.

Financial or Other Competing Interests: None.

\section{REFERENCES}

[1] Sonawane R. Abetalipoproteinemia. Pediatr Oncall J 2016;13(1):1-8.

[2] Isa HM, Mohamed AM. Abetalipoproteinemia: three case reports, a novel microsomal triglyceride transfer protein gene mutation and a literature review. J Clin Case Rep 2016;6(9):1-5.

[3] Bassen FA, Kornzweig AL. Malformation of the erythrocytes in a case of atypical retinitis pigmentosa. Blood 1950;5(4):381-7.

[4] Uslu N, Gurakan F, Yuce A, et al. Abetalipoproteinemia in an infant with severe clinical phenotype and a novel mutation. The Turkish Journal of Paediatrics 2010;52(1):73-7.

[5] Zamel R, Khan R, Pollex RL, et al. Abetalipoproteinemia two case reports and literature review. Orphanet J Rare Dis 2008;3:19. 